\title{
A Note on Rational Approximation
}

\section{By Robert W. Floyd}

It is suggested by plausible reasoning and confirmed by experience that the error of an $n$th degree polynomial approximation, in the Chebyshev sense of least maximum error, to an analytic function, is roughly a multiple of the $n+1$ st Chebyshev polynomial, $T_{n+1}(x)$, on the interval of approximation. Therefore if the $n$th degree polynomial $f^{*}(x)$ is equal to the function, $f(x)$, on the roots of $T_{n+1}(x)$, we expect that $f^{*}(x)$ will be a satisfactory approach to a Chebyshev approximation of $f(x)$.

Because $f(x)$ is analytic, it may be represented with negligible error in the interval of approximation by a polynomial $p(x)$ of sufficiently high degree; e.g., a truncated Taylor's or Maclaurin's series. Applying the division algorithm for polynomials,

$$
\begin{aligned}
& p(x)=q_{0}(x) \cdot T_{n+1}(x)+r_{0}(x) \\
& T_{n+1}(x)=q_{1}(x) \cdot r_{0}(x)+r_{1}(x) \\
& r_{0}(x)=q_{2}(x) \cdot r_{1}(x)+r_{2}(x) \\
& r_{1}(x)=q_{3}(x) \cdot r_{2}(x)+r_{3}(x), \text { etc., }
\end{aligned}
$$

where the degrees of the $r_{i}$ form a strictly decreasing sequence. From these equations we may wite $r_{i}(x)=a_{i}(x) \cdot p(x)+b_{i}(x) \cdot T_{n+1}(x)$, where $a_{i}$ and $b_{i}$ are defined recursively by

$$
\begin{array}{lll}
a_{i}=a_{i-2}-q_{i} \cdot a_{i-1}, & a_{-1}=0, & a_{-2}=1 \\
b_{i}=b_{i-2}-q_{i} \cdot b_{i-1}, & b_{-1}=1, & b_{-2}=0 .
\end{array}
$$

It may be proven that the sum of the degrees of $a_{i}(x)$ and $r_{i}(x)$ is at most $n$. The first set of e(puations may be written $p(x)=\left[r_{i}(x) / a_{i}(x)\right]-\left[b_{i}(x) / a_{i}(x)\right] \cdot T_{n+1}(x)$, so that $r_{i}(x) / a_{\imath}(x)$ is a rational approximation to $p(x)$, exact wherever $T_{n+1}(x)$ vanishes. Since $T_{n+1}(x) \leqq 1$ in the interval of approximation, $b_{i}(x) / a_{i}(x)$ provides a bound for the error of the approximation. If $b_{i}(x) / a_{2}(x)$ is nearly constant on the interval of approximation, the error oscillates between $n+2$ extrema of nearly equal magnitude, and the method of approximation is justified, for Chebyshev approximation is characterized by an error which oscillates at least $n+1$ times between positive and negative extrema of equal magnitude. For the particular case $i=0, a_{i}=1$, and $r_{0}(x)$ is a polynomial approximation to $f(x)$ of degree at most $n$.

For example; $f(x)=e^{x}=1+x+\left(x^{2} / 2 !\right)+\left(x^{3} / 3 !\right)+\cdots$;

$$
\begin{aligned}
p(x)=1+x+ & .5 x^{2}+.16666667 x^{3}+.04166667 x^{4}+.00833333 x^{5} \\
& +.00138889 x^{6}+.00019841 x^{7}+.00002480 x^{8}+.00000276 x^{9} .
\end{aligned}
$$

For $-1 \leqq x \leqq 1,|p(x)-f(x)| \leqq 3.0 \times 10^{-i} . T_{i}(x)=64 x^{7}-112 x^{5}+56 x^{3}-7 x$. Then $q_{0}=\left(317.5625+38.75 x+4.3125 x^{2}\right) \times 10^{-3}$;

Received July 6, 1959. 
$r_{0}=1+1.00002223 x+.50000271 x^{2}+.16648913 x^{3}+.04164497 x^{4}$

$+.00868659 x^{5}+.00143229 x^{6}$

$$
\left|p(x)-r_{0}\right|=\left|q_{0}\right| \cdot\left|T_{7}(x)\right| \leqq 3.61 \times 10^{-6} \quad(-1 \leqq x \leqq 1) \text {. }
$$

Therefore $\left|f(x)-r_{0}\right| \leqq 3.91 \times 10^{-6}(-1 \leqq x \leqq 1)$. Dividing $T_{7}(x)$ by $r_{0}$, $q_{1}=-270,998.81+44,683.688 x$.

$r_{1}=270,998.81+226,314.15 x+90,815,458 x^{2}+22,832.391 x^{3}$

$$
+3,846.3890 x^{4}+381.2048 x^{5} \text {. }
$$

$a_{0}=1 ; b_{0}=-q_{0}$

$a_{1}=-q_{1} ; b_{1}=1+q_{1} q_{0}$

Therefore

$$
p(x)=\frac{r_{1}}{a_{1}}-\frac{b_{1}}{a_{1}} T_{7}=-\frac{r_{1}}{q_{1}}+\frac{1+q_{1} q_{0}}{q_{1}} T_{7}(x) .
$$

The second term on the right is

$$
\frac{.13940940+.036886598 x+.0056281054 x^{2}+.0019269840 x^{3}}{-270,998.81+44,683.688 x} T_{7}(x)
$$

whose absolute value is bounded by $8.121 \times 10^{-7}$ for $-1 \leqq x \leqq 1$. Thus $e^{x}$ may be approximated on this interval by

$$
-\frac{r_{1}}{q_{1}}=\frac{\begin{array}{c}
1+.83511123 x+.33511386 x^{2}+.08425274 x^{3} \\
+.01419338 x^{4}+.00140667 x^{5}
\end{array}}{1-.16488518 x}
$$

where the error is bounded by $\pm\left(3 \times 10^{-7}+8.1 \times 10^{-7}\right)= \pm 1.1 \times 10^{-6}$.

Armour Research Foundation

Illinois Institute of Technology

Chicago 16, Illinois

1. C. Hastinas, Approximations for Digital Computers, Princeton, 1955, p. 47-64.

2. F. B. HIDDEBRAND, Introduction to Numerical Analysis, McGraw-Hill Book Co., New York, 1956, p. 389-395.

3. NBS APPLed Mathematics Series, 9, Tables of Chebyshev Polynomials $S_{n}(x)$ and $C_{n}(x)$, U. S. Govt. Printing Office, Washington, D. C., 1952, p. 16-18.

\section{The Complete Factorization of $2^{132}+1$}

\section{By K. R. Isemanger}

The integer $2^{132}+1$ is divisible by $2^{44}+1=17 \cdot 353 \cdot 2931542417$ and the quotient, $2^{88}-2^{44}+1$, is divisible by $241 \cdot 7393$. There remains the formidable problem of factoring the resultant quotient $N$, where $N$ is the integer

173700820402235083057.

Received July 3, 1959; in revised form, September 17, 1959. 